\title{
AN UNLABELED BRACKETING SOLUTION TO THE PROBLEM OF CONJOINED PHRASES \\ IN MONTAGUE'S PTQ*
}

\section{INTRODUCTION}

In The proper treatment of quantification in ordinary English (: $\cdot \mathrm{rQ}$ ) (Montague, 1973), Richard Montague presents the syntax and semantics of a fragment of English. His objective is to support the contention that "there is ... no important theoretical difference between natural languages and the artificial languages of logicians" and that it is "possible to comprehend the syntax given in PTQ consists of an inductive definition for each non-empty mathematically precise theory" [Montague, 1970, p. 373]. The formal syntax given in PTQ consists of an inductive definition for each non-empty grammatical category, in particular, the category of sentences. Parallel rules give a translation into an intensional logic for which semantics are provided.

The semantic aspects of PTQ, particularly the elegance of the syntax/ semantics relation, are impressive. The range of English expressions generated by the syntax of PTQ is limited, but the fragment was selected for inclusion of philosophically difficult sentences. The standard logical operators have English representatives in Montague's syntax; and by the translation rules for the fragment, these English words are explicitly correlated with the corresponding logical operators. The propositional operators are represented by and's of sentences and of verb phrases, and or's of sentences, verb phrases, and term phrases, and by negation of verb phrases; quantifiers are represented by every, the, and $a$; the modal operator for necessity by necessarily; and tense operators by the future and present perfect tenses of verbs. In addition, the syntax includes pronouns and allows relative clauses (formed with such that). Although the syntax of PTQ is weak, especially by current transformational standards, the work is important enough to justify investigating how far the fragment can be extended toward English.

In this paper we approach this task by asking whether the system of PTQ can be modified so that the fragment which it claims to handle is actually handled correctly. The system as presented by Montague allows conjunction and disjunction of intransitive verb phrases and disjunction of term phrases. 
We first explore the relation of these operations with the rest of the system and show that they are not properly integrated.

Partee (1973) observes that the verb phrase agreement rule (S4), which joins a subject with a verb phrase to form a sentence, incorrectly inflects only the first verb in a conjoined verb phrase, producing John talks and walk in lieu of John talks and walks. Bennett (1976) points out that there are also problems with term phrase disjunctions such as he $\mathbf{f}_{1}$ or he h $_{2}$ in rules using $F_{10, n}$ (substitution) or $F_{5}$ (accusative formation), and remarks that any accommodation of disjunctive terms would involve considerable complexity. But these are only a few of the problems induced by conjunction in PTQ. There are other types of sentences that are strictly ungrammatical, and some which, while grammatical, do not correspond to their translations into logic.

Problems occur with every syntax rule that takes as argument a conjoined intransitive verb phrase or term phrase and forms its result by any process other than concatenation of the unmodified arguments. That is, informally, all agreement rules fail for conjunctions and disjunctions.

The problem is not restricted to the syntactic aspects of PTQ, but threatens the integrity of the system, since it extends to the relation of the syntax and the semantics. For example, the syntactically correct sentences John seeks Mary or Bill and John finds her and John seeks Bill or Mary and John finds him are, on one reading, synonymous, with the pronouns coreferential with the disjunctive term phrases.

The primary difficulty is traced to the fact that agreement operators consistently modify the first item of a category when they should modify more than one. We propose a correction that utilizes unlabeled bracketing of constituents. The bracketing makes possible a recursive definition of a set of first items and leads to a natural and satisfactory solution to the problems noted, Technical details of this change are presented below.

This solution contrasts with the two solutions previously proposed, both of which considered conjoined verbs only. Partee (1973) proposes a labeled bracketing. Bennett (1976) uses markers to distinguish uninflected forms of verbs. Another strong mechanism that might be used to solve the conjunction problems is one introduced by Thomason (1976). He includes structure by allowing rules on 'analyzed expressions', where an analyzed expression is a phrase together with its analysis tree.

For the convenience of the reader, the syntax rules of PTQ, with brackets added but otherwise unchanged, are given in an Appendix. 


\section{THE SEMANTIC ROLE OF CONJUNCTION IN PTQ}

It is important to note the semantic role of conjunctions in PTQ. At first glance it might appear that the motivation for conjoined phrases was purely syntactic, since, for example, John or Bill walks and John walks or Bill walks have translations that are logically equivalent. However, as we examine how the conjoined phrases fit into other constructions, we see that there are meanings that are obtained using $\mathrm{S} 11$ (sentence conjunction), S1 2 (intransitive verb phrase conjunction), and S13 (term phrase conjunction) that cannot otherwise be obtained. Thus, John and Bill will walk would not have the same interpretation as John will walk and Bill will walk. In this section we discuss the semantic independence of the conjunction and disjunction rules. For this section only, we include term-phrase conjunction as well as disjunction, since Montague's reasons for excluding it would appear to be purely syntactic.

\section{II.1. Criteria for rule independence}

To be able to argue that a particular conjunction rule is essential to PTQ, we must first have a notion of rule independence in a system. We describe several possible criteria and select one of them on the basis of its appropriateness to a semantic-syntactic system like PTQ.

Syntactic independence of a rule $S$ in a set of rules means simply that there is a sentence $\phi$ that cannot be derived without using rule $S$. That is, if rule $S$ is deleted, the language itself is changed. The three conjunction rules in PTQ are clearly syntactically independent. This is shown by the following sentences: (S11-and) John runs and Bill walks, (S11-or) John runs or Bill walks, (S12-and) John runs and walks, (S12-or) John runs or walks, (S13-and) John and Bill run, (S13-or) John or Bill runs. Note that if PTQ were to be augmented by conjunction-reduction transformations, the $\mathrm{S} 12$ and $S 13$ examples could be derived using $S 11$. Notice also that two of the most interesting rules of PTQ, S15 and S16, are unnecessary by this criterion.

Sentence-semantic independence of a rule $S$ can be defined as: some sentence $\phi$ has a meaning by $S$ that is not logically equivalent to any meaning for $\phi$ that is obtained without $S$. That is, if rule $S$ is deleted, some sentence has fewer meanings. Partee $(1975 ;$ p. 236) argues that $S 15$ is not 'superfluous in PTQ' by giving a sentence that shows that $\mathbf{S} 15$ is sentencesemantic independent. 
Syntactic independence of rule $S$ implies sentence-semantic independence of rule $S$. For, if a syntactically independent $S$ is deleted, a sentence which had at least one meaning now has no derivations and hence no meanings. Therefore, the conjunction rules also meet the criterion of sentencesemantic independence in PTQ.

Are the conjunction rules semantically independent?

Semantic independence of a rule $S$ in a set of rules can be defined as: some sentence $\phi$ has a meaning by $S$ that is not logically equivalent to any meaning obtained without $S$ for any sentence $\psi$. That is, if $S$ is deleted, some meaning is lost. Semantic independence implies sentence-semantic independence; however, no relation of implication holds between semantic independence and syntactic independence.

Suppose we were to add conjunction-reduction transformations to PTQ so that S12 and S13 were no longer syntactically independent. Then for the augmented system the criteria of sentence-semantic independence and semantic independence of $\mathrm{S} 12$ and $\mathrm{S} 13$ would be the same. This is what Bennett (1974; p. 115) does in discussing whether disjunctive terms might be 'not essential'. He adds the conjunction-reduction rules and then uses the criterion of sentence-semantic independence. However, Bennett's counterexample is for his extension of PTQ and falls outside PTQ proper.

We now consider the semantic independence of rules in PTQ proper. Clearly, S11-and and S11-or are independent in PTQ by this criterion too. We are able to show that S12-and and S13-and are semantically independent in PTQ. For S12-and the sentence John hasn't talked and walked has as one of its meaning $7 H\left[\operatorname{talk}^{\prime}(\hat{\jmath}) \wedge\right.$ walk' $\left.\left({ }^{\wedge} j\right)\right]$. No meaning equivalent to this can be obtained without S12-and. For S13-and, the sentence John and Bill will walk has a meaning $W\left[\right.$ walk $^{\prime}\left({ }^{\wedge} j\right) \wedge$ walk $\left({ }^{\wedge} b\right]$ not obtainable without this rule. S12-or and S13-or are not semantically independent in PTQ. The difference here results from the fact that $H$ and $W$ are both essentially existential quantifiers on time variables. Therefore they distribute over disjunction but not over conjunction. The formula $H \phi \vee H \psi$ is logically equivalent to $H[\phi \vee \psi]$, but $H \phi \wedge H \psi$ is not equivalent to $H[\phi \wedge \psi]$.

In both of the examples, $\mathrm{S} 17$ was used. S17 is peculiar in that it adds the sentence operators, $H, W$, and $ᄀ$, as it forms the sentence, rather than applying them to previously constructed sentences. In PTQ minus S17, we are unable to find such examples. The necessity operator acis as a universal quantifier over points of reference. Since syntactically it applies to 
sentences, the distributed and undistributed forms can always be obtained. Attempts to construct examples using the fact that the ordinary universal quantifier distributes over $\wedge$ but not over $\vee$ will fail because the quantifi cation rules make it possible to get the undistributed meaning whenever it is possible to get the distributed one. Thus, $(\Lambda x)[\phi \vee \psi]$ can be obtained by $S 14$, even though it cannot be obtained by factoring the universal quantifier in $(\Lambda x) \phi \vee(\Lambda x) \psi$.

\section{CONJUNCTION RELATEDERRORS IN PTQ}

In this section we examine the rules of PTQ in detail, locating those which lead to syntactic or semantic errors when used with conjoined phrases. Each syntactic rule of PTQ defines the categories of its arguments and of its result, and uses an operator $F_{i}$ (or operator schema $F_{i, n}$ ) to form the result. The rules that fail do so because they do not take into account the fact that the argument might be a conjoined phrase. For each operator that can take as argument a conjoined phrase we look for examples that suggest necessary changes.

In PTQ the only items that may be conjoined or disjoined are intransitive verb phrases, term phrases (disjunction only), and sentences. We first examine verb phrases, then term phrases. Sentence conjunctions and disjunctions do not have the problems noted here.

\section{III.1. Verb phrases}

Conjunction and disjunction of intransitive verb phrases lead to syntactic errors by both of the rules that join subject and verb phrase to form a sentence. No allowance has been made for agreement of any but the first verb.

Verb agreement rule. As Partee (1973) has pointed out, the operator $F_{4}$ of rule $\mathrm{S4}$, which forms a sentence by concatenating a term phrase with an inflected intransitive verb phrase, fails for conjoined intransitive verb phrases because only the first verb is replaced by its third person singular. Thus, PTQ generates Mary loves John or love Bill and the man runs or change. $F_{4}$ cannot be corrected by replacing all verbs by their third person singular because, for example, from the man and try to change the result the man tries to change is correct. The operator must know and take into account the difference in structure between try to change and run or change. Thus, to correct $F_{4}$ we must replace the modification of 'the first 
verb' by modification of 'the first verbs', which we define below using unlabeled bracketing.

Rules of tense and sign. Like rule S4, rule S17 forms sentences by concatenating a term phrase with an intransitive verb phrase in which 'the first verb' has been replaced by an inflected form. The five parts of S17 call for replacement by the negative third person singular present $\left(F_{11}\right)$, the third person singular future $\left(F_{12}\right)$, the negative third person singular future $\left(F_{13}\right)$, the third person singular present perfect $\left(F_{14}\right)$, and the negative third person singular present perfect $\left(F_{15}\right)$. As we would now expect, $S 17$ produces improper results for conjoined verb phrases. $F_{14}$ yields, for example, John has talked and walk and $F_{15}$ yields John hasn't talked and walk. The results of $F_{11}$ : John doesn't talk and walk, $F_{12}$ : John will talk and walk, and $F_{13}$ : John won't talk and walk, are correct, because no change in talk and walk is needed.

Correction of $\$ 17$ will also need syntactic structure, but it will not be as straightforward as that for S4. As we will argue later, an examination of the corresponding logical translation shows that the result for $F_{15}$ should be John hasn't talked and walked, not John hasn't talked and hasn't walked. Thus we cannot simply replace each verb by its inflected form as in rule S4, but must insert the auxiliary in front of the first verb only. We note that the syntax does not produce sentences with conjoined verb phrases that are different in tense. Presumably John has talked and has walked would be added in the course of whatever extension to the system produces John has talked and will walk.

Before suggesting restatements of S4 and S17, we consider the syntactic and semantic errors that arise from term phrase disjunction.

\section{III.2. Term phrases}

Montague limits compound term phrases to disjunctions in order to avoid introducing plural verbs. Syntactic and semantic errors caused by term phrase disjunction appear (1) when pronoun agreement in gender is required with a disjoined term phrase used as argument to $F_{10, n}$ (rules $\mathrm{S} 14, \mathrm{~S} 15$, $S 16$ ), and (2) when a pronoun he $e_{k}$ occurs as a disjunct of a term phrase used as an argument to $F_{5}$ or $F_{10, n}$ (S5, S6, S14, S15, S16).

Pronoun agreement. The rules of quantification, S14, S15, and S16, use the operator schema $F_{10, n}$ to substitute a term phrase for pronouns he ${ }_{n}$ and $\operatorname{him}_{n}$. The operator is defined so that the first such pronoun is replaced by 
the term phrase and each later occurrence of he $_{n}$ or him $_{n}$ is replaced by the singular pronoun of the gender of the first noun in the term phrase and of the case (nominative or accusative) of the he or him $_{n}$ replaced. This works properly if the term phrase is not a disjunction. If it is a disjunction, agreement is to the first disjunct only, and the result is syntactically well-formed, but does not correspond to the intended semantic interpretation.

Our examples show that this error arises for pronouns in either subject or object position.

Example. The two sentences (1) John or Mary finds a fish and he eats it, and (2) Mary or John finds a fish and she eats it, are both syntactically correct and PTQ produces them both with their correct interpretations. That is, there is a derivation of (1) in which he and John are coreferential and one of (2) in which she and Mary are coreferential. However, both (1) and (2) are ambiguous in PTQ and on one pair of readings they are synonymous. From the sentence he ${ }_{0}$ finds a fish and he ${ }_{0}$ eats it, rule S14 using $F_{10,0}$ produces (1) by substituting the term phrase John or Mary for he $\mathbf{e}_{0}$, and produces (2) by substituting Mary or John for he $e_{0}$. Thus, in (1) he and John or Mary are coreferential, and in (2) she and Mary or John are coreferential. For these readings, the sentences have translations that are logically equivalent.

The error arises because by $F_{10, n}$ the pronoun agrees in gender with the 'first $B_{C N}$ or $B_{T}$ ' in the substituted phrase. The results should be something like John or Mary finds a fish and he or she eats it, and Mary or John finds a fish and she or he eats it. However, a rule that yieids these results will also give John or Bill finds a fish and he or he eats it, and other even less acceptable sentences.

We might try to avoid this syntactic difficulty by not allowing conjoined term phrases to be substituted in by the rules of quantification. This doesn't eliminate the problem because the subrule is not syntactically independent: any sentence obtainable by substituting a conjoined term phrase for a variable has an alternative derivation in which the term phrases are substituted separately into a conjunction of variables. For example, the last sample sentence above can be obtained by substituting John for he ${ }_{0}$ and Bill for $h_{1}$ in he $_{0}$ or he $e_{1}$ finds a fish and he or he $_{1}$ eats it.

Furthermore, the subrule is sentence-semantic independent in PTQ. The sentence Mary doesn't love Bill or John has two meanings: $7($ love' $(m, b) v$ love, $(m, j))$ and ᄀlove', $_{*}(m, b) \vee$ ᄀlove' $_{*}(m, j)$. To obtain the latter meaning 
the conjoined term phrase Bill or John must be substituted into a sentence by quantification rule $\mathrm{S} 14$.

Example. As an example of the failure of $F_{10, n}$ for pronouns in object position, consider the sentence John seeks him hand find(s) him $_{3}$, and the two term phrases Mary or Bill and Bill or Mary. The results of substituting these term phrases for him ${ }_{3}$ by $F_{10,3}$ are respectively John seeks Mary or Bill and find(s) her, and John seeks Bill or Mary and find(s) him. Derived in this way the sentences have logically equivalent translations. The sentence John seeks Mary or Bill and find(s) her or him is also derivable and synonymous with the other two.

Substitution of disjoined pronouns. We have shown that $F_{10, n}$ fails for replacement of pronouns by disjoined term phrases. An additional error occurs in $F_{10, n}$ and also in the operator $F_{5}$ of rules S5 and S6. Each of these operators is used with one argument a term phrase, and each has two alternatives depending on whether the term phrase has the form he ${ }_{n}$. They give no special consideration to the case in which the term phrase does not have the form he ${ }_{n}$, but does contain such a pronoun as a disjunct.

For example, $F_{5}$ in rule $\mathrm{S} 5$ yields correctly seek John and seek him ${ }_{4}$ for the term phrases John and he $\mathbf{H}_{4}$, but also yields seek John or he ${ }_{4}$ instead of seek John or him 4 for the term phrase John or he $4 . F_{5}$ in rule S6 gives correctly about him ${ }_{2}$ and about Mary, but gives about he ${ }_{2}$ or Mary for the term phrase he ${ }_{2}$ or Mary.

In $F_{10, n}$ this error produces, for example, Mary loves he or he $_{2}$ from Mary loves him ${ }_{3}$ because the rule substitutes he ${ }_{1}$ or he ${ }_{2}$ unchanged for the first occurrence of he ${ }_{3}$ or him $_{3}$. It also yields Bill seeks John and Bill finds he or Mary, from Bill seeks him ${ }_{0}$ and Bill finds him ${\text {. First, by } F_{10,1} \text {, him }}_{1}$ can be replaced by he $e_{0}$ or Mary to give Bill seeks himo and Bill finds he h $_{0}$ or Mary. A correct application of S14, this time using $F_{10,0}$, gives Bill seeks John and Bill finds he or Mary.

For completeness, we note that the corrections needed for $F_{10, n}$ in rule S14 are also needed in S15 and S16, because a sentence can be embedded in a common noun phrase or in an intransitive verb phrase.

It is obvious that to revise the functions so that they replace all the he ${ }_{n}$ occurring in the term phrase by corresponding him $_{n}$ does not provide a correction, since the grammatical case of he $e_{0}$ must be preserved in the generation by $F_{5}$ of about a man such that he ${ }_{0}$ finds him. The examples make clear that it is necessary to consider the form of all top level term phrases in a disjoined term phrase. 


\section{PRELIMINARY DEFINITIONS}

We now begin a proposal to modify the rules of PTQ. In this section we develop some definitions that will then allow us to rewrite the rules.

\section{IV.1. Discussion}

Need for constituent structure. In her analysis of the error in the verb agreement rule Partee (1973) points out that the two sentences John tries to walk and talks and John tries to walk and talk are counterexamples to any attempt to repair the system without using brackets for syntactic structure. She sug. gests that if the rule operates on labeled bracketings it can distinguish

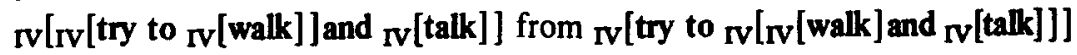
as required.

As Partee's example makes clear, any correction must employ some knowledge of constituent structure to identify the items to be modified when a phrase is embedded in another construction. In the example, the bracketing allows the correct choice of try and talk in the first sentence, and try only in the second, as the verbs to be inflected when the intransitive verb phrase is concatenated after a term phrase to form a sentence.

An unlabeled bracketing. For our purposes an unlabeled bracketing will be used, although, of course, a labeled one would work too. The rules of PTQ, bracketed but otherwise unchanged, are given in the Appendix. For Partee's example the bracketings are [[[try to]walk] and talk] and [[try to] [walk and talk] ]. This is sufficient to allow us to determine that in the first case there are two top level verb phrases [ [try to] walk] and talk, and hence two first verbs to be inflected, and that in the second there is only one top level verb phrase, the whole phrase itself, and hence only one first verb, try.

\section{IV.2. Bracketing Conventions}

The rules given in the Appendix are bracketed according to the following conventions:

(i) A basic expression containing more than one word is bracketed, e.g., [assert that]; the basic expressions he $e_{0}$, he $_{1}, \ldots$ are replaced by the bracketed expressions [he 0 ], [he 1 ], . . ; a word constructed by adding a suffix to a basic expression is bracketed, e.g., [run s], [walk ed] are used as the inflected forms called for by rules $\mathrm{S} 4$ and $\mathrm{S} 17$. (Except for readability, it would be preferable to bracket every basic expression. Indeed, if a labeled 
bracketing were used, this would be necessary. None of the algorithms given below would change if every basic expression were bracketed.)

(ii) Expressions formed by concatenation operators $F_{0}, F_{1}, F_{2}$ are bracketed, e.g., [every man]; expressions formed by concatenation of two arguments, possibly with modification of one of them (by $F_{5}, F_{6}$, and $F_{7}$ ) are bracketed.

(iii) Relative clause expressions, formed by $F_{3, n}(\zeta, \phi)$, are bracketed as

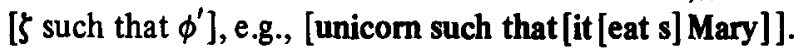

(iv) Sentences formed by concatenating a term with an intransitive verb phrase modified for tense, number, or sign, $\left(F_{4}, F_{11}, F_{12}, F_{13}, F_{14}, F_{15}\right)$ are bracketed.

(v) Conjunctions $F_{8}(\alpha, \beta)$ and $F_{9}(\alpha, \beta)$ are bracketed as $[\alpha$ and $\beta]$, $[\alpha$ or $\beta]$.

(vi) No additional bracketing is introduced by the pronoun replacement operators $F_{10, n}$.

Examples of sentences bracketed by these rules are:

[necessarily [[Mary or ninety] [[[seek s] [John or it]] or [[find s] [ninety or[he 1]]]]]], [[[Mary or ninety] or Mary] [talk s]], and [John hasn't [walk ed]].

\section{IV.3. First Phrases}

For PTQ unlabeled bracketing suffices to identify the top level conjuncts and disjuncts of an intransitive verb phrase or of a term phrase. Given these top level elements it is then possible to identify the verbs to be inflected in a verb phrase (the first verbs) and the nouns and variables for agreement in a term phrase (the first nouns in first term phrases, the first variables). The rules of PTQ can then be restated to refer to the set of first verbs, rather than to the first verb, for example.

We define first the set of first term phrases of a term phrase and the set of first intransitive verb phrases of an intransitive verb phrase.

A phrase is member of $P_{A}$ for some category $A$. Phrases correspond to the balanced bracketed expressions of the revised grammar: Every basic phrase is balanced since each operation $F_{i}$ forms a new phrase by embedding balanced expressions within an outer pair of brackets.

First term phrases. A term phrase is any member of $\boldsymbol{P}_{T}$. The set of first term phrases of a term phrase $\gamma$ is defined recursively: If $\gamma$ is of the form 
[ $\alpha$ or $\beta$ ], where $\alpha$ and $\beta$ are phrases, then the first term phrases of $\gamma$ are the first term phrases of $\alpha$ and of $\beta$; otherwise, $\gamma$ is the first term phrase of $\gamma$.

First variables [he $n$ ]. The first variables [he $n$ ] of a term phrase $\gamma$ are the variables [he $n$ ] that are first term phrases of $\gamma$.

First intransitive verb phrases. An intrasitive verb phrase is any member of $P_{\mathrm{IV}}$. The set of first intransitive verb phrases of an intransitive verb phrase $\gamma$ is defined recursively: If $\gamma$ is of the form [ $\alpha$ or $\beta$ ], or [ $\alpha$ and $\beta$ ], where $\alpha$ and $\beta$ are phrases, then the first intransitive verb phrases of $\alpha$ and of $\beta$ are first intransitive verb phrases of $\gamma$; otherwise, $\gamma$ is the first intransitive verb phrase of $\boldsymbol{\gamma}$.

First verbs. A verb is any member of $B_{\mathrm{IV}}, B_{\mathrm{TV}}, B_{\mathrm{IV} / \mathrm{t}}$, or $B_{\mathrm{IV} / \mathrm{IV} \text {. }}$ (Notice that [try to] and [believe that] are counted as verbs, contrary to ordinary usage.) The first verbs of an intransitive verb phrase $\gamma$ are the leftmost verbs of the members of the set of first intransitive verb phrases of $\gamma$.

Examples. The intransitive verb phrase [[seek[John or it]] or[find ninety]] has two first phrases [seek [John or it]] and [find ninety]. Its first verbs are the first verbs of those phrases, that is, seek and find. The first verbs of [[[try to] talk] and walk] are the first verbs of [[try to] talk] and of walk, hence, [try to] and walk.

The definition of first verbs depends on the particular rules for formation of intransitive verb phrases in PTQ, namely, S1, S5, S7, S8, S10, S12, and S16.

First verbs and first words. It is an artifact of PTQ, though not a fact of English, that the first word (basic expression) in an intransitive verb phrase is always the verb. An alternative definition equivalent to the one above is thus: the first verbs of an intransitive verb phrase $\gamma$ are the left-most basic expressions of the members of the set of first intransitive verb phrases of $\boldsymbol{\gamma}$.

A basic requirement in order to be able to give a definition of first verbs is that verbs be identifiable in some way. In PTQ they are identifiable both by position and by membership in the fixed basic sets. If PTQ produced, for example, [John [allegedly [run s]]], the alternative definition based on first word would fail. The definition based on first verb will work as long as lexical ambiguity between verbs and other basic expressions is not introduced. PTQ has no lexical ambiguity, although, as Thomason [1974, p. 11 fn.] points out, Montague does not in general rule it out of his disambiguated languages.

Transformational extension. Partee (1973) argues that the necessity of labeled bracketing in rule S4 provides independent justification for the 
labeled brackets needed if transformations are introduced into the system. Since, as we will show, unlabeled bracketing suffices for $\$ 4$, the argument is really the other way around: since labeled bracketing will be needed for transformations, it might well be introduced in the basic rules.

\section{MODIFICATION OF PTQRULES}

Revised statements of parts of PTQ can now be given. It is important to note that the simplicity of statement is possible only because we use the previously given recursive definitions of first verbs, first variables, and first term phrases.

\section{V.1. Verb phrases}

Verb agreement rule. A corrected statement of the verb agreement rule can now be given:

If $\alpha \in P_{T}$ and $\delta \in P_{\mathrm{IV}}$, then $F_{4}^{\prime}(\alpha, \delta) \in P_{t}$, where $F_{4}^{\prime}(\alpha, \delta)=$ $\left[\alpha \delta^{\prime}\right]$ and $\delta^{\prime}$ is the result of replacing each first verb in $\delta$ by its third person singular present.

The previous syntactic difficulty is now removed. $F_{4}$ is used only in S4, so its redefinition does not create any new problems. It is easy to verify that the corresponding translation rule $\mathrm{T} 4$ is unaffected by the change.

Operators for tense and negation. It was noted earlier that the operators of rule $\mathrm{S} 17$ cannot be revised by strict analogy to the change to $F_{4}$, because the result, while syntactically correct would not be compatible with the semantics for S17. We now expand on that.

The formal translation of John has talked and walk is equivalent to $H\left[\right.$ talk $_{*}{ }^{\prime}(j) \wedge$ walk $\left._{*}{ }^{\prime}(j)\right]$. John talks translates to talk ${ }_{*}{ }^{\prime}(j)$ and John walks to walk ${ }_{*}^{\prime}(j)$. If we examine the interpretation for the intensional logic we find that $H \phi$ is to be counted as true at the moment of time $j$ if and only if for some moment of time $j^{\prime}$ prior to $j, \phi$ is true. Thus, $H(\phi \wedge \psi)$ is true only if $\phi$ and $\psi$ have been simultaneously true. The English sentence which is closest to having this meaning is then the desired result of $F_{14}$. Accordingly, we should rewrite $F_{14}$ to produce [John[has [[talk ed] and [walk ed]]]]. This decision to have only one occurrence of the auxiliary is confirmed by examination of the other four operators of $\mathrm{S} 17$ and by considering disjunction of verb phrases as well as conjunction. 
The evidence is strongest for the negative present perfect operator, $F_{15}$, applied to a disjunctive verb phrase, e.g., John hasn't talked or walked. The translation for the sentence formed by concatenating John with the negative present perfect of talk or walk is $7 H\left[\right.$ talk $_{*}{ }^{\prime}(j) \vee$ walk $\left._{*}{ }^{\prime}(j)\right]$. This translates back into English as "It is not the case that at any previous time John talked or walked", which is equivalent to "John hasn't talked or walked", i.e., "John hasn't talked and hasn't walked", but not to "John hasn't talked or hasn't walked".

This leads us to rewrite the operators of $\mathrm{S17}$ so that they put the (negated) auxiliary before the conjunction. $S 17$ becomes $S 17$ ':

$$
\begin{aligned}
& \text { If } \alpha \in P_{\mathrm{T}} \text { and } \delta \in P_{\mathrm{IV}}, \text { then } F_{11}^{\prime}(\alpha, \delta), F_{12}^{\prime}(\alpha, \delta), \\
& F_{13}^{\prime}(\alpha, \delta), F_{14}^{\prime}(\alpha, \delta), \text { and } F_{15}^{\prime}(\alpha, \delta) \in P_{\mathrm{t}}, \text { where } \\
& F_{11}^{\prime}(\alpha, \delta)=[\alpha \text { doesn't } \delta], \\
& F_{12}^{\prime}(\alpha, \delta)=[\alpha \text { will } \delta], \\
& F_{13}^{\prime}(\alpha, \delta)=[\alpha \text { won't } \delta], \\
& F_{14}^{\prime}(\alpha, \delta)=\left[\alpha \text { has } \delta^{\prime}\right], \text { and } \delta^{\prime} \text { is the result of replacing each } \\
& \text { first verb in } \delta \text { by its past participle, } \\
& F_{15}^{\prime}(\alpha, \delta)=\left[\alpha \text { hasn't } \delta^{\prime}\right], \text { and } \delta^{\prime} \text { is as above. }
\end{aligned}
$$

\section{V.2. Term phrases}

Just as correction for conjoined verb phrases requires the definition of 'first verbs', so correction for disjoined term phrases requires the definitions of 'first variables' and 'first term phrases'. We begin with $F_{5}$ because it is ałsó a subcase of $F_{10, n}$.

Correction of $F_{5} . F_{5}$ is used to form an intransitive verb phrase or a prepositional phrase by concatenating respectively a transitive verb or a preposition with a tem phrase that has been put into the accusative case. Montague's statement of $F_{5}$ fails because it considers only two alternatives for the term phrase, according as it has or does not have the form he $\mathbf{e}_{n}$. This would be correct except that term phrases may be disjunctions in which he ${ }_{n}$ occur, e.g., the term phrase the man such that he ${ }_{2}$ loves him or he $e_{3}$, which becomes accusative by replacing he ${ }_{3}$ by him $_{3} . F_{5}$ must be revised to take structure into account and to replace exactly those variables he $e_{n}$ that are at the top levels of the term phrase. These are the 'first variables he ${ }_{n}$ ' defined above. The statement becomes $F_{5}^{\prime}(\delta, \beta)=\left[\delta, \beta^{\prime}\right]$, and $\beta^{\prime}$ is the result of replacing each first variable he ${ }_{n}$ in $\beta$ by $\operatorname{him}_{n}$. 
Correction of $F_{10, n}$. The operator $F_{10, n}$ substitutes a representative of a term phrase for an occurrence of a variable. The variable occurrence may be in the nominative (he ${ }_{n}$ ) or in the accusative $\left(\mathrm{him}_{n}\right)$, and may be the first occurrence of a variable for this $n$ or a later occurrence. There are thus four cases, and the operator $F_{10, n}$ must behave differently for each. An example with all four cases is

\section{[[[he 3][[find s] [him 1]]] or [[he 1] won't [lose [him 3]]]].}

[he 3] is a first-nominative occurrence and [him 3] a later-accusative occurrence; [him 1] is a first-accusative occurrence and [he 1] is later-nominative occurrence. If the term phrase [John or [he 5]] is substituted by $F_{10,3}$ for [he 3] and [him 3], and [Bill or [he 7]] is substituted by $F_{10,1}$ for [he 1] and [him 1], we should obtain [[[John or [he 5]][[find s] [Bill or [him 7]]]] or [[he or [he 7]] won't [lose [him or [him 5]]]]], where each term phrase is obtained in a slightly different way.

Using the concepts of first variables and first term phrases the four cases can be stated:

$F_{10, n}^{\prime}(\alpha, \phi)$ comes from $\phi$ by replacing the first occurrence of either he ${ }_{n}$ or him $\operatorname{hin}_{n}$ by if he ${ }_{n}$ and by $\alpha^{\prime}$ if $\operatorname{him}_{n}$, and replacing later occurrences of $h_{n}$ by $\alpha^{\prime \prime}$ and later occurrences of him $m_{n}$ by $\alpha^{\prime \prime}$, where $\alpha^{\prime}$ is the result of replacing each first variable he $e_{k}$ in $\alpha$ by him ${ }_{k} ; \alpha^{\prime \prime}$ is the result of replacing each first term phrase in $\alpha$ not of the form he $k_{k}$ by $\left\{\begin{array}{l}\text { he } \\ \text { she } \\ \text { it }\end{array}\right\}$ according as the gender of the first $B_{\mathrm{CN}}$ or $B_{\mathrm{T}}$ in the term phrase is $\left(\begin{array}{l}\text { masc. } \\ \text { fem. } \\ \text { neuter }\end{array}\right)$; and $\alpha^{\prime \prime \prime}$ is the result of replacing each first variable he $e_{k}$ in $\alpha$ by $\operatorname{him}_{k}$ and replacing each of the other first term phrases in $\alpha$ by $\left\{\begin{array}{l}\text { him } \\ \text { her } \\ \text { it }\end{array}\right\}$ according as the gender of the first $B_{\mathrm{CN}}$ or $B_{\mathrm{T}}$ in the term phrase is $\left\{\begin{array}{l}\text { masc. } \\ \text { fem. } \\ \text { neuter }\end{array}\right\}$.

Note that in the example the substitutions were $\alpha$ and $\alpha^{m}$ for $F_{10,3}^{\prime}$ and $\alpha^{\prime}$ and $\alpha^{\prime \prime}$ for $F_{10,1}^{\prime}$. 


\section{SUMMARY OF MODIFICATIONSTOSYNTACTIC RULES}

We can summarize the modifications to the syntactic rules as follows: The basic rules $\mathrm{S1}, \mathrm{S} 2$, and $\mathrm{S} 3$ are unchanged. For the rules of functional application, in $\mathrm{S} 4, \mathrm{~S} 5$, and $\mathrm{S} 6$, the operator $F_{4}$ is replaced by $F_{4}^{\prime} ;$ rules $\mathrm{S} 7$, $\mathrm{S} 8, \mathrm{~S} 9$, and $\mathrm{S} 10$ are unchanged. In the rules of quantification, $\mathrm{S} 14, \mathrm{~S} 15$, an S16, the operator schema $F_{10, n}$ is replaced by $F_{10, n}^{\prime}$. In the rule of tense and sign, $\mathrm{S} 17$, the operators $F_{11}, \ldots, F_{15}$ are replaced by $F_{11}^{\prime}, \ldots, F_{15}^{\prime}$, respectively.

\section{CONCLUSIONS}

Although Montague claims that the system of The proper treatment of quantification in ordinary English includes some conjunction and disjunction, the rules for other grammatical constructions do not take conjunction or disjunction into account, and in general fail either syntactically or semantically when one of their arguments is so formed. Using an unlabeled bracketing of syntactic structure and recursive definitions, we have been able to rewrite the rules so that correct results are obtained.

These results should provide a firmer basis for extension of PTQ.

\section{University of Michigan}

\section{APPENDIX.BRACKETED PTQ SYNTAX RULES}

The rules of this Appendix are those of PTQ except that he $\boldsymbol{e}_{i}$ has been replaced by [he $i$ ] throughout, and brackets have been added.

Basic Expressions

$$
\begin{array}{ll}
B_{\mathrm{IV}}= & \text { \{run, walk, talk, rise, change } \\
B_{\mathrm{T}}= & \text { \{John, Mary, Bill, ninety, [he } 0],[\text { he } 1], \\
& [\text { he } 2], \ldots\} \\
B_{\mathrm{TV}}= & \{\text { find, lose, eat, love, be, seek, conceive } \\
B_{\mathrm{IAV}}= & \text { \{rapidly, slowly, voluntarily, allegedly }\} \\
B_{\mathrm{CN}}= & \text { \{man, woman, park, fish, pen, unicom, price, } \\
& \text { temperature }
\end{array}
$$




$$
\begin{aligned}
& B_{\mathrm{IAV} / \mathrm{T}}=\{\text { in, about }\} \\
& B_{\mathrm{IV} / \mathrm{t}}=\{[\text { believe that], [assert that] }\} \\
& B_{\mathrm{IV} / / \mathrm{IV}}=\{[\text { try to], [wish to] }\}
\end{aligned}
$$

\section{Syntactic Rules}

\section{Basic Rules}

S1. $\quad \boldsymbol{B}_{\boldsymbol{A}} \subseteq \boldsymbol{P}_{\boldsymbol{A}}$ for every category $\boldsymbol{A}$.

S2. If $\zeta \in P_{\mathrm{CN}}$, then $F_{0}(\zeta), F_{1}(\zeta), F_{2}(\zeta) \in P_{\mathrm{T}}$,

where $F_{0}(\zeta)=$ [every $\zeta$ ],

$F_{1}(\zeta)=$ [the $\left.\zeta\right]$,

$F_{2}(\zeta)$ is [a $\left.\zeta\right]$ or [an $\left.\zeta\right]$ according as the first word in $\zeta$ takes a or an.

S3. If $\zeta \in P_{\mathrm{CN}}$ and $\phi \in P_{t}$, then $F_{3, n}(\zeta, \phi) \in P_{\mathrm{CN}}$,

where $F_{3, n}(\zeta, \phi)=\left[\zeta\right.$ such that $\left.\phi^{\prime}\right]$, and $\phi^{\prime}$ comes from $\phi$ by replacing each occurrence of he or $_{n}$ him $_{n}$ by $\left(\begin{array}{l}\text { he } \\ \text { she } \\ \text { it }\end{array}\right)$ or $\left(\begin{array}{l}\text { him } \\ \text { her } \\ \text { it }\end{array}\right)$ respectively, according as the first $B_{\mathrm{CN}}$ in $\zeta$ is of $\left(\begin{array}{l}\text { masc. } \\ \text { fem. } \\ \text { neuter }\end{array}\right)$ gender.

\section{Rules of functional application}

S4. If $\alpha \in P_{\mathrm{T}}$ and $\zeta \in P_{\mathrm{IV}}$, then $F_{4}(\alpha, \delta) \in P_{\mathrm{t}}$, where $F_{4}(\alpha, \delta)=\left[\alpha \delta^{\prime}\right]$ and $\delta^{\prime}$ is the result of replacing the first verb (i.e., member of $B_{\mathrm{TV}}, B_{\mathrm{TV}}, B_{\mathrm{rV} / \mathrm{t}}$, or $B_{\mathrm{IV} / / \mathrm{IV}}$ ) in $\delta$ by its third person singular present.

S5. If $\delta \in P_{\mathrm{rV} / \mathrm{T}}$ and $\beta \in P_{\mathrm{T}}$, then $F_{\mathrm{s}}(\delta, \beta) \in P_{\mathrm{IV}}$, where $F_{5}(\delta, \beta)=[\delta \beta]$ if $\beta$ does not have the form [he $\left.n\right]$ and $F_{5}(\delta,[$ he $n])=[\delta[\operatorname{him} n]]$.

S6. If $\delta \in P_{\mathrm{IAV} / \mathrm{T}}$ and $\beta \in P_{\mathrm{T}}$, then $F_{5}(\delta, \beta) \in P_{\mathrm{IAV}}$. 
57. If $\delta \in P_{\mathrm{IV} / \mathrm{t}}$ and $\beta \in P_{t}$, then $F_{6}(\delta, \beta) \in P_{\mathrm{IV}}$, where $F_{6}(\delta, \beta)=[\delta \beta]$.

S8. If $\delta \in P_{\mathrm{IV} / \mathrm{IV}}$ and $\beta \in P_{\mathrm{IV}}$, then $F_{6}(\delta, \beta) \in P_{\mathrm{IV}}$.

S9. If $\delta \in P_{\mathrm{t} / \mathrm{t}}$ and $\beta \in P_{\mathrm{t}}$, then $F_{6}(\delta, \beta) \in P_{\mathrm{t}}$.

S10. If $\delta \in P_{\mathrm{IV} / \mathrm{TV}}$ and $\beta \in P_{\mathrm{IV}}$, then $F_{7}(\delta, \beta) \in P_{\mathrm{IV}}$, where $F_{7}(\delta, \beta)=[\beta \delta]$.

\section{Rules of conjunction and disyunction}

S11. If $\phi, \psi \in P_{t}$, then $F_{8}(\phi, \psi), F_{9}(\phi, \psi) \in P_{t}$,

$$
\text { where } \begin{aligned}
F_{8}(\phi, \psi) & =[\phi \text { and } \psi], \\
F_{9}(\phi, \psi) & =[\phi \text { or } \psi] .
\end{aligned}
$$

S12. If $\gamma, \delta \in P_{\mathrm{IV}}$, then $F_{8}(\gamma, \delta), F_{9}(\gamma, \delta) \in P_{\mathrm{IV}}$.

S13. If $\alpha, \beta \in P_{\mathrm{T}}$, then $F_{9}(\alpha, \beta) \in P_{\mathrm{T}}$.

\section{Rules of quantification}

S14. If $\alpha \in P_{\mathrm{T}}$ and $\phi \in P_{\mathrm{t}}$, then $F_{10, n}(\alpha, \phi) \in P_{\mathrm{t}}$,

where either (i) $\alpha$ does not have the form [he $k$ ], and $F_{10, n}(\alpha, \phi)$ comes from $\phi$ by replacing the first occurrence of [he $n$ ] or [him $n$ ] by $\alpha$ and all other occurrences of [he $n$ ] or [him $n]$ by $\left\{\begin{array}{l}\text { he } \\ \text { she } \\ \text { it }\end{array}\right\}$ or $\left\{\begin{array}{l}\text { him } \\ \text { her } \\ \text { it }\end{array}\right\}$ respectively, according as the gender of the first $B_{\mathrm{CN}}$ or $B_{\mathrm{T}}$ in $\alpha$ is $\left\{\begin{array}{l}\text { masc. } \\ \text { fem. } \\ \text { neuter }\end{array}\right\}$, or (ii)

$\alpha=$ [he $k$ ], and $F_{10, n}(\alpha, \phi)$ comes from $\phi$ by replacing all occurrences of [he $n$ ] or [him $n$ ] by [he $k$ ] or [him $k$ ] respectively.

S15. If $\alpha \in P_{T}$ and $\zeta \in P_{\mathrm{CN}}$, then $F_{10, n}(\alpha, \zeta) \in P_{\mathrm{CN}}$.

S16. If $\alpha \in P_{\mathrm{T}}$ and $\delta \in P_{\mathrm{IV}}$, then $F_{10, n}(\alpha, \delta) \in P_{\mathrm{IV}}$.

Rules of tense and sign

S17. If $\alpha \in P_{\mathrm{T}}$ and $\delta \in P_{\mathrm{IV}}$, then $F_{11}(\alpha, \delta), F_{12}(\alpha, \delta), F_{13}(\alpha, \delta)$, 
$F_{14}(\alpha, \delta), F_{15}(\alpha, \delta) \in P_{t}$, where $F_{11}(\alpha, \delta)=\left[\alpha \delta^{\prime}\right]$ and $\delta^{\prime}$ is the result of replacing the first verb in $\delta$ by its negative third person singular present; $F_{12}(\alpha, \delta)=\left[\alpha \delta^{\prime \prime}\right]$ and $\delta^{\prime \prime}$ is the result of replacing the first verb in $\delta$ by its third person singular future; $F_{13}(\alpha, \delta)=\left[\alpha \delta^{\prime \prime \prime}\right]$ and $\delta^{\prime \prime \prime}$ is the result of replacing the first verb in $\delta$ by its negative third person singular future; $F_{14}(\alpha, \delta)=\left[\alpha \delta^{\prime \prime \prime \prime}\right]$ and $\delta^{\prime \prime \prime \prime}$ is the result of replacing the first verb in $\delta$ by its third person singular present perfect; $F_{15}(\alpha, \delta)=\left[\alpha \delta^{\prime \prime \prime \prime}\right]$ and $\delta^{\prime m m}$ is the result of replacing the first verb in $\delta$ by its negative third person singular present perfect.

\section{NOTES}

* This research was supported in part by NSF Grants GS-31309 and BNS 76-23840. Our observations were made with the aid of several computer programs for PTQ: a sentence generator for the bracketed syntax, a parser, and a translator. I should like to thank Philip Tedeschi, David Warren, and David Lewis for helpful discussions. Warren is also acknowledged as co-author of the section on the semantic role of conjunction.

\section{B I B LI O G R A P HY}

Michael Bennett, Some Extensions of a Montague Fragment of English, Ph.D. Dissertation, University of California, Los Angeles, 1974.

Michael Bennett, in Partee (ed.), A Variation and Extension of a Montague Fragment of English, 1976, pp. 119-163.

J. Hintikka, J. Moravcsik, and P. Suppes (eds.), Approaches to Natural Language, Reidel, Dordrecht, 1973.

M. LaGaly, R. Fox, and A. Bruck (eds.), Papers from the Tenth Regional Meeting of the Chicago Linguistic Society, University of Chicago, Chicago, 1974.

Richard Montague, 'English as a formal language', (EFL), in Bruno Visentini, et al., Linguaggi nella Societa e nella Tecnica, Edizioni di Comunita, Milan, 1970 , pp. 198-224; reprinted in Montague, 1974, pp. 188-221.

Richard Montague, in Hintikka et al., The Proper Treatment of Quantification in Ordinary English, (PTQ), 1973, pp. 221-242; reprinted in Montague, 1974, 247-270.

Richard Montague, Formal Philosophy: Selected Papers of Richard Montague, edited and with an introduction by Richmond Thomason, Yale University Press, New Haven, 1974.

Barbara Partee, 'Some Transformational Extensions of Montague Grammar', J. Philosophical Logic 2 (1973), 509-534; reprinted in Partee (ed.), 1976, 51-76.

Barbara Partee, 'Montague Grammar and Transformational Grammar', Linguistic Inquiry VI (1975), 203-300. 
Barbara H. Partee (ed.), Montague Grammar, Academic Press, New York, 1976.

Richmond H. Thomason, in LaGaly et al., Some Complement Constructions in Montague Grammar, 1974, pp. 712-722.

Richmond H. Thomason, in Partee (ed.), Some Extensions of Montague Grammar, 1976, pp. 77-117. 